\title{
A comparison of the effectiveness of four aerosol shielding devices in reducing endoscopists' exposure to airborne particles during simulated upper gastrointestinal endoscopy
}

\author{
Akira Higashimori, Yuji Nadatani, Hirotsugu Maruyama, Yasuhiro Fujiwara \\ Department of Gastroenterology, Osaka City University Graduate School of Medicine, Osaka, Japan
}

The coronavirus disease-2019 (COVID-19) pandemic has highlighted the importance of reducing the risk of infection through aerosol generation. Aerosol particles $\geq 5 \mu \mathrm{m}$ and $<5 \mu \mathrm{m}$ in diameter are referred to as droplets and airborne particles, respectively. Since upper gastrointestinal (GI) endoscopy is an aerosol-generating procedure, ${ }^{1}$ several novel devices have been proposed to reduce the exposure of the endoscopist to aerosol particles. ${ }^{2-5}$ However, there is insufficient scientific evidence for the efficacy of these devices. While some of these devices have been examined using simulated cough-producing fluorescent droplets, ${ }^{2,5}$ these simulations did not evaluate small invisible droplets or airborne particles.

Using an in situ simulation model, four proposed devices (modified mask [Fig. 1A], vinyl box with and without continuous suction [Figs. 1B, C], aerosol box [Fig. 1D], and no device [control]) were compared in terms of the exposure of the endoscopist to airborne particles. The simulation was performed in a self-contained endoscopy room with nine room air changes per hour. Upper GI endoscopy was performed by six endoscopists on a mannequin with a mouth guard, using a 9.9-mm flexible video GI scope (GIF-H290; Olympus Japan Limited, Tokyo,

Received: May 31, 2021 Revised: July 14, $2021 \quad$ Accepted: July 20, 2021 Correspondence: Akira Higashimori

Department of Gastroenterology, Osaka City University Graduate School of Medicine, 1-4-3, Asahimachi Abeno-ku, Osaka 545-8585, Japan

E-mail: higamo@med.osaka-cu.ac.jp

(a) This is an Open Access article distributed under the terms of the Creative Commons Attribution Non-Commercial License (http://creativecommons.org/licenses/by-nc/4.0/) which permits unrestricted non-commercial use, distribution, and reproduction in any medium, provided the original work is properly cited.
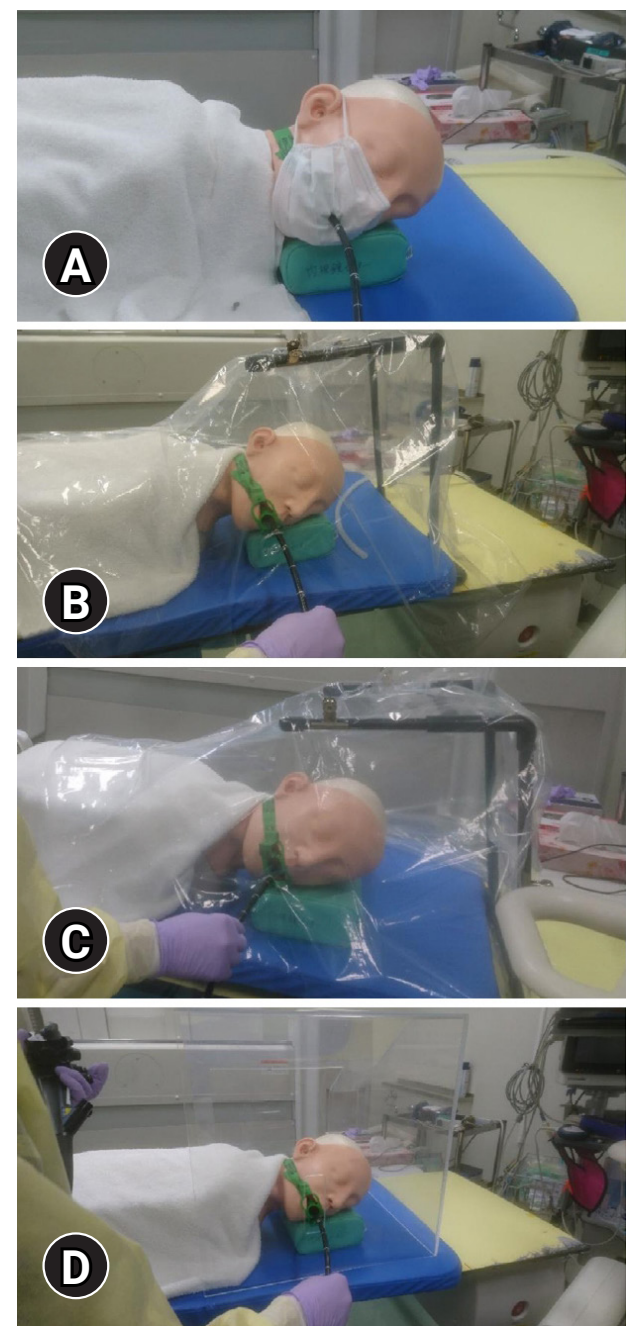

Fig. 1. Overview photographs for tested devices. (A) Modified mask. (B) Vinyl box with continuous suction. (C) Vinyl box without suction. (D) Aerosol box. 
Japan). To simulate a strong cough, saline was sprayed via a $0.4-\mathrm{MPa}$ pressure atomizer nozzle, placed in the mannequin's hypopharynx..$^{5-7}$ Simulated upper GI endoscopy was performed for five minutes on a mannequin. After device removal, the airborne particles were counted for one minute. A cough was generated every 30 seconds using a spray containing saline. The exposure of the endoscopist to airborne particles $(0.3-2 \mu \mathrm{m})$ was measured using a portable HHPC6+ handheld particle counter (Beckman Coulter Inc., Brea, CA, USA). A detailed description of the methods is provided in the Supplementary Material 1.

The total airborne amount during the simulation is shown in Figure 2, as well as in Table 1. The modified mask significantly reduced the total amount of airborne particles. The vinyl box with and without suction reduced the total amount of airborne particles, and using the suction significantly increased its effectiveness. There was no significant difference in the total amount of airborne particles between the aerosol box and control. Separate analyses for different particle sizes $(0.3,0.5,1$, and $2 \mu \mathrm{m})$ yielded similar results as the total airborne particles (Supplementary Material 2).

In this study, both the modified mask and vinyl box significantly reduced the exposure of the endoscopist to the total amount of airborne particles. In contrast, the aerosol box was ineffective. Furthermore, the adjunctive use of continuous suction with the vinyl box resulted in a significantly lower amount of airborne particles than vinyl box use without suction. To the best of our knowledge, no prior studies have used an in situ simulation model to compare the effectiveness of various aerosol shielding devices in reducing endoscopist exposure to airborne particles during endoscopy.

The modified mask and vinyl box with continuous suction exhibited the most potent protective effect against exposure to airborne particles while maintaining the airborne particle amount at baseline levels. Among the tested devices, the modified mask provided the optimal coverage and closest adaptation to the mannequin's mouth. Its effectiveness highlighted the importance of sealing and covering the point of aerosol generation to minimize endoscopist exposure to airborne particles. Furthermore, the modified mask was the most simple, inexpensive, and easily disposable device among the tested masks. Its simple design also minimized the risk of interference with the endoscopic procedure. Therefore, the modified mask was the most practical, effective, and economical method to reduce endoscopist exposure to aerosol particles during the COVID-19 pandemic.

The aerosol box was the most commonly proposed aerosol shielding device. However, it provided no additional benefits compared to endoscopy without the use of a shielding device. Our results were similar to those of Simpson et al., who recently reported that the use of an aerosol box provided no
Intervention
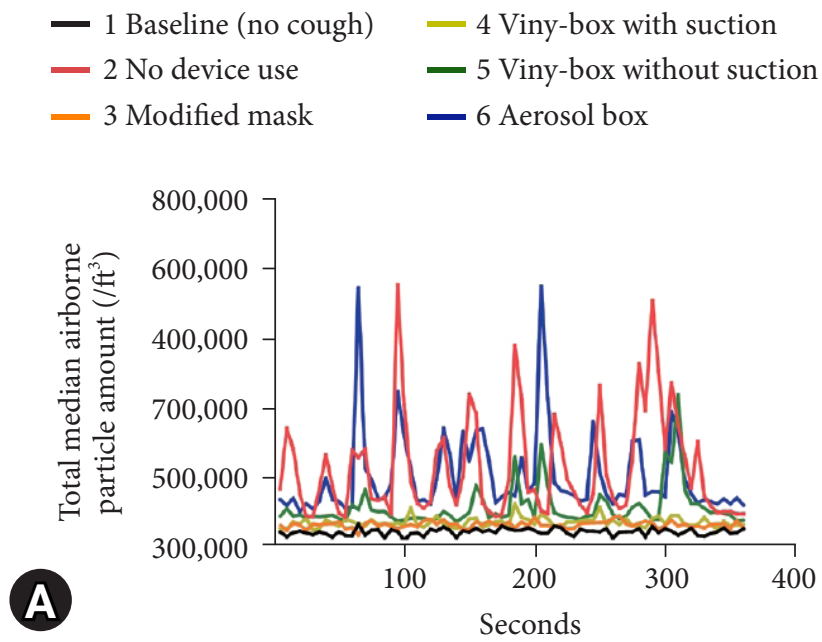

Intervention

- Baseline (no cough) $\quad \Delta$ Viny-box with suction

- No device use

- Modified mask

$\checkmark$ Viny-box without suction

- Aerosol box

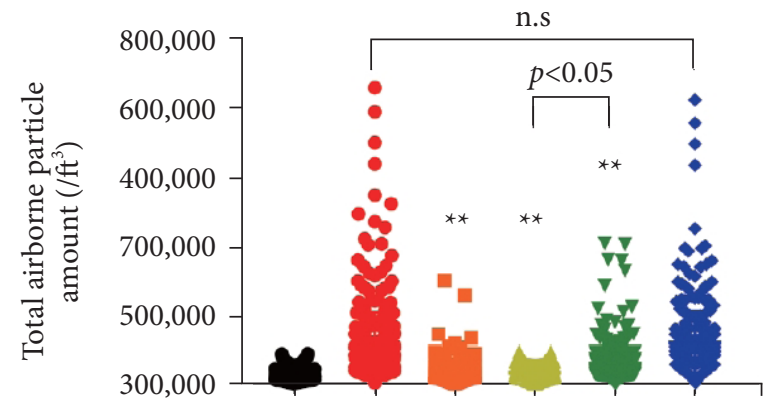

B

Fig. 2. Comparison of the total amount of airborne particles among the different experimental conditions. (A) Time series chart of each intervention over the 6 min experimental period. Lines represent the median total particle count $(0.3-2 \mu \mathrm{m})$ of six separate trials. Coughs were generated every 30 seconds through the experimental period. (B) The number of total airborne particles during the simulation is shown on a scatter plot with medians and interquartile ranges. $n=432 .{ }^{* *} p<0.01$ (versus no device use). n.s, not significant. 


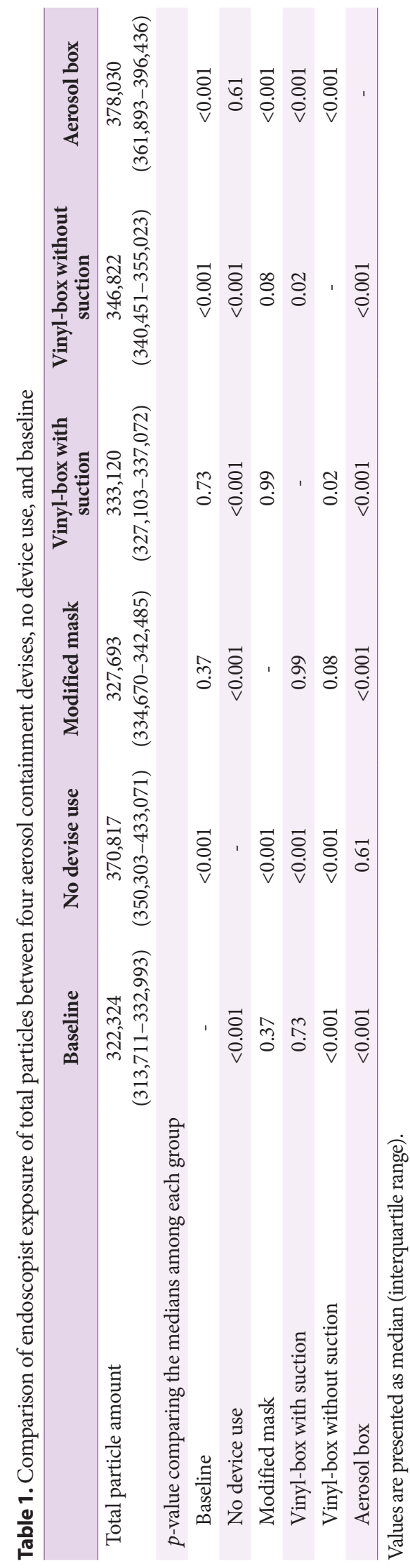

protective effects against airborne particle exposure during simulated tracheal intubation. Dalli et al. ${ }^{9}$ demonstrated that a significant amount of aerosol escaped from the arm access hole during simulated coughing. Although these studies involved simulated tracheal intubation, similar effects may be expected during endoscopic procedures. Aerosolized particle clouds escaped from the open side of the aerosol box during each simulated cough. Thus, shielding devices should be designed with smaller openings to reduce endoscopist exposure to airborne particles.

The vinyl box had a smaller opening than the aerosol box. This reduced the exposure to airborne particles. Continuous suction provided a significant benefit. The United States Food and Drug Administration recently issued an alert stating that the use of protective barrier enclosures without negative pressure increased the risk of COVID-19 infection in healthcare providers. ${ }^{10}$ Our results supported the use of devices, such as the vinyl box, which had continuous suction to create a negative pressure effect within protective barriers.

Our study had several limitations. First, our simulation partially reproduced the actual endoscopy conditions. Further studies in clinical settings are needed to validate the protective effects of these aerosol shielding devices, especially those of modified masks and vinyl boxes (with continuous suction), against COVID-19 infection. Second, an increase in the quantity of airborne particles did not necessarily equate to a rise in viral infectivity during endoscopy. Despite numerous studies on the transmission routes of respiratory viruses, the relationship between these two variables remains vague. Third, the role of the upper GI tract in the transmission of severe acute respiratory syndrome coronavirus 2 remains unclear. Fourth, the devices evaluated in this study required the endoscopist to use personal protective equipment while performing endoscopic examinations. However, our results showed that some of these devices could contain almost all the generated airborne particles during simulated endoscopy. This suggested that using these devices allow endoscopists to perform endoscopy more safely than when using personal protective equipment alone.

In conclusion, this study demonstrated that the modified masks and devices with continuous suction effectively reduced endoscopist exposure to airborne particles during upper GI endoscopy. In contrast, both aerosol boxes and vinyl boxes (without suction) were ineffective. 


\section{Supplementary Material}

Supplementary Material 1. Supplementary material and methods.

Supplementary Material 2. Post-hoc comparisons of endoscopic exposure to each particle size under different experimental conditions.

Supplementary materials related to this article can be found online at https://doi.org/10.5946/ce.2021.175.

\section{Conflicts of Interest}

The authors have no potential conflicts of interest.

\section{Funding}

None.

\section{Author Contributions}

Conceptualization: AH, YN, HM, YF; Data curation: AH, YN, HM; Formal analysis: AH; Supervision: YN, HM, YF; Writing-original draft : AH; Writing-review \& editing: AH, YN, HM, YF.

\section{ORCID}

Akira Higashimori Yuji Nadatani Hirotsugu Maruyama Yasuhiro Fujiwara

https://orcid.org/0000-0002-9950-9899 https://orcid.org/0000-0001-7081-8746 https://orcid.org/0000-0002-8728-8101 https://orcid.org/0000-0002-1225-1785

\section{REFERENCES}

1. Chan SM, Ma TW, Chong MK-C, et al. A proof of concept study: esophagogastroduodenoscopy is an aerosol-generating procedure and continuous oral suction during the procedure reduces the amount of aerosol generated. Gastroenterology 2020;159:1949-1951.
2. Sagami R, Nishikiori H, Sato T, et al. Endoscopic shield: barrier enclosure during the endoscopy to prevent aerosol droplets during the COVID-19 pandemic. VideoGIE 2020;5:445-448.

3. Kobara H, Nishiyama N, Masaki T. Shielding for patients using a single-use vinyl-box under continuous aerosol suction to minimize SARS-CoV-2 transmission during emergency endoscopy. Dig Endosc 2020;32:e114-e115.

4. Endo H, Koike T, Masamune A. Novel device for preventing diffusion of aerosol droplets from subjects undergoing esophagogastroduodenoscopy during COVID-19 pandemic. Dig Endosc 2020;32:e140-e141.

5. Maruyama H, Higashimori A, Yamamoto K, et al. Coronavirus disease outbreak: a simple infection prevention measure using a surgical mask during endoscopy. Endoscopy 2020;52:E461-E462.

6. Canelli R, Connor CW, Gonzalez M, et al. Barrier enclosure during endotracheal intubation. N Engl J Med 2020;382:1957-1958.

7. Gupta JK, Lin CH, Chen Q. Flow dynamics and characterization of a cough. Indoor Air 2009;19:517-525.

8. Simpson JP, Wong DN, Verco L, et al. Measurement of airborne particle exposure during simulated tracheal intubation using various proposed aerosol containment devices during the COVID-19 pandemic. Anaesthesia 2020;75:1587-1595.

9. Dalli J, Khan MF, Marsh B, et al. Evaluating intubation boxes for airway management. Br J Anaesth 2020;125:e293-e295.

10. U.S. Food and Drug Administration (FDA). Protective barrier enclosures without negative pressure used during the COVID-19 pandemic may increase risk to patients and health care providers: letter to health care providers [Internet]. Silver Spring (MD): FDA; 2020 [updated 2020 Aug 21; cited 2021 Aug 4]. Available from: https://www. fda.gov/medical-devices/letters-health-care-providers/protective-barrier-enclosures-without-negative-pressure-used-during-covid-19-pandemic-may-increase. 